\title{
Características Morfológicas de un Grupo de Zarigüeyas (Didelphys marsupialis) del Suroccidente Colombiano
}

\author{
Morphological Characteristics of a Group of Opossums (Didelphys \\ marsupialis) IN SOUTHWESTERN COLOMBIA
}

\author{
Santiago Lozada ${ }^{1}$, Ginés Fernando Ramírez ${ }^{1}$, José Henry Osorio ${ }^{2,3}$
}

\section{Resumen}

La familia Didelphidae representa la mayoría de los marsupiales americanos ampliamente distribuidos en América del Sur, ocupando casi todo tipo de hábitats, a excepción de zonas altas y zonas desérticas. Los marsupiales de la familia varían de tamaño y algunos autores los describen como medianos y grandes, mientras que otros los describen de tamaño pequeño y mediano. El presente trabajo aporta una descripción morfológica de las zarigüeyas ubicadas en una región del suroccidente colombiano.

Palabras clave: zarigüeya, morfología, taxonomía, mamíferos

\section{Abstract}

Didelphidae family represents the majority of American marsupials distributed in South America. They are present in most type of habitats except in high mountains and deserts. They have different sizes and are described by some authors as medium and large size, while others indicate small and medium sizes. The present work contributes with a description of Didelphys marsupialis located in the south west region of Colombia

Key words: Didelphidae, morphology, taxonomy, mammals

\footnotetext{
${ }^{1}$ Departamento de Salud Animal, ${ }^{2}$ Laboratorio de Bioquímica Clínica y Patología Molecular, Departamento de Ciencias Básicas de la Salud, Universidad de Caldas, Manizales, Colombia

${ }^{3}$ E-mail:jose.osorio_o@ucaldas.edu.co
}

Recibido: 8 de febrero de 2014

Aceptado para publicación: 25 de noviembre de 2014 


\section{INTRODUCCIÓN}

La zarigüeya fue el primer marsupial conocido en Europa a principios del siglo XVl, cuando fue llevado un ejemplar desde Brasil a España y observaron el saco que poseía en su abdomen (marsupium «pequeña bolsa»). En 1758, este mamífero fue clasificado por Linnaeus como Didelphis marsupialis, perteneciente al orden de los insectívoros (Grimwood, 1969). Actualmente, los didelfimorfos (Didelphimorphia) son un orden de mamíferos que agrupa a la mayor parte de los marsupiales e incluye 92 especies, todas en la familia Didelphidae (Wison y Reeder, 2005).

Los miembros de la familia Didelphidae presentan la cabeza de forma cónica y de tamaño grande en relación con su cuerpo, un hocico puntiagudo de nariz desnuda, ojos grandes y separados y orejas redondeadas, de aspecto membranoso y desprovistas de pelo (Emmons y Feer, 1990). Sus miembros son cortos y cada pata presenta cinco dedos, donde el primer dedo de los miembros posteriores es oponible, característica que les permite sujetar objetos y mejorar su habilidad trepadora (Vonhof, 2000). En la mayoría de los géneros, la cola es de tipo prensil (Stein y Patton, 2008). Algunos géneros no presentan el marsupio (Marmosa, Monodelphis, Metachirus) o solo presentan un par de pliegues laterales (Solari et al., 2001), en tanto que se encuentra bien desarrollado en los géneros Didelphis, Philander y Chironectes (Patton et al., 2000). La fórmula dentaria es I 5/4, C 1/1, P 3/3, M 4/4, con una característica de interés para determinar la edad ya que su tercer molar carece de raíz en la etapa juvenil (Gardner y Creighton, 2008).

Son omnívoros (frutas maduras, vegetales, hojas, néctar, flores, invertebrados, pequeños vertebrados) y tienen hábitos mayormente nocturnos. El periodo de gestación es de 12 a 15 días, después de los cuales las crías pasan al marsupio por 60 a 70 días (Vaughan et al., 1999).
La diversidad de este grupo de mamíferos es tan amplia que es imposible reunirlos a todos en esta única entidad ordinal (Oliviera y Goin, 2006). Por esta razón, no se ha llegado a un consenso definitivo sobre la clasificación taxonómica de este grupo, pese al avanzado desarrollo de las técnicas morfológicas, bioquímicas y cariológicas (Marques-Aguilar, 2008). El presente trabajo aporta una descripción morfológica de las zarigüeyas ubicadas en una región del suroccidente colombiano, lo que permite tener un mayor conocimiento de esta especie, amenazada por el crecimiento de las poblaciones urbanas.

\section{Materiales y Métodos}

El estudio se realizó en los predios del Zoológico de Cali, ubicado en la vertiente oriental de la Cordillera Occidental, a las márgenes del río Cali, Municipio de Santiago de Cali, departamento de Valle del Cauca, Colombia. La posición geográfica del zoológico es $3^{\circ} 27^{\prime} 01^{\prime \prime} \mathrm{N}$; $76^{\circ} 33^{\prime} 35.8^{\prime \prime} \mathrm{W}$; presenta una superficie de 25 ha y se encuentra ubicado al oeste de la ciudad, a una altitud de $1013 \mathrm{msnm}$.

El clima de la región, según el Instituto de Hidrología, Meteorología y Estudios Ambientales (IDEAM), es templado seco, con una temperatura promedio de $24.8^{\circ} \mathrm{C}$ y una humedad relativa entre 75 y $80 \%$. El promedio de precipitación pluvial anual es de $1169 \mathrm{~mm}$, con un periodo de baja precipitación a principios de año y en julio y agosto, y otro de alta precipitación en abril-mayo y octubre-noviembre. El paisaje se describe como bosque seco neotropical, donde la vegetación se encuentra representada por plantas ornamentales y algunos relictos de bosque secundario subandino de galería.

Se siguió la técnica de muestreo recomendada por Alvares et al. (2006). La captura de las zarigüeyas se realizó en un periodo de 12 semanas (del 10 de diciembre de 
Cuadro 1. Zarigüeyas capturadas $(\mathrm{n}=15)$ en los predios del Zoológico de Cali, según su edad, sexo, peso y longitud total

\begin{tabular}{cccccccc}
\hline \multirow{2}{*}{ Parámetros biológicos } & \multicolumn{2}{c}{$\begin{array}{c}\text { Zarigüeyas } \\
\text { capturadas }\end{array}$} & \multicolumn{2}{c}{ Peso $(\mathrm{g})$} & \multicolumn{2}{c}{ Longitud total (mm) } \\
\cline { 3 - 8 } & $\mathrm{n}$ & $\%$ & Promedio & d.e. & Promedio & d.e. \\
\hline \multirow{3}{*}{ Edad } & Adulto & 8 & 53 & 2000 & 169 & 839 & 52 \\
& Pre-adulto & 3 & 20 & 1697 & 150 & 792 & 103 \\
& Juvenil & 4 & 27 & 650 & 286 & 644 & 128 \\
\multirow{3}{*}{ Sexo } & Macho & 8 & 53 & 2120 & 113 & 844 & 21 \\
& Hembra & 7 & 47 & 1924 & 163 & 836 & 67 \\
\hline
\end{tabular}

2007 al 1 de marzo de 2008). El muestreo se organizó repartiendo el área en 12 parcelas de $1000 \mathrm{~m}^{2}$ aproximadamente, muestreando una a una hasta completar las 12 ha del parque. El recorrido comenzó desde la zona oriental hasta la zona occidental. El área se dividió en tres zonas ( $\mathrm{A}, \mathrm{B}$ y $\mathrm{C}$ ), donde la zona A correspondió a la parte administrativa del parque y fue dividida en 2 parcelas; la zona $\mathrm{B}$ correspondió a la parte de exhibición y se dividió en siete parcelas; la zona $\mathrm{C}$ correspondió a la Unidad de Bienestar Animal (UBA) y se dividió en tres parcelas.

Se empleó el método de captura de Sánchez (2004). Cada área se muestreó durante cinco días y se emplearon cuatro trampas tipo Tomahawk $(30 \times 30 \times 60 \mathrm{~cm})$ y dos trampas tipo Sherman $(11 \times 12 \times 30 \mathrm{~cm})$ cebadas con pollo. Se ubicaron en el suelo y se colocaron en supuestas rutas de paso de los marsupiales (vegetación, fuentes de agua y alimento) y en posibles sitios de refugio y anidación. Se muestrearon 72 puntos, capturándose 15 zarigüeyas.

Los animales fueron pesados en una balanza digital Ohaus DS4 con capacidad para $20 \mathrm{~kg}$, y se anestesiaron con una dosis bolo de xilazina $(2.2 \mathrm{mg} / \mathrm{kg})$ y clorhidrato de ketamina (Imalgene $\left.{ }^{\circledR}\right)(30 \mathrm{mg} / \mathrm{kg})$, vía intramuscular. Se hizo la identificación taxonómica empleando las claves para el orden Didelphimorfia (Cuartas y Muñoz, 2003). Se identificó el sexo y se tomaron las medidas corporales de longitud total, de la cola, del pie y de la oreja con un calibrador digital Mitutoyo con precisión de $0.01 \mathrm{~mm}$. La edad fue calculada mediante la técnica de fórmula dental (Schweigmann et al., 1999), que consiste en observar la erupción y desgaste dentario del maxilar superior. Se colocó un tatuaje en la oreja derecha a los animales capturados a fin de evitar la toma de información en caso volvieran a caer en las trampas.

\section{Resultados y Discusión}

Se capturaron 15 individuos de la especie Didelphys marsupialis (Cuadro 1). Hubo capturas en todas las zonas del parque, encontrándose un individuo cada 0.8 ha. Una zarigüeya se capturó en un techo y el resto fue en el suelo.

Teniendo en cuenta los parámetros biológicos de edad, sexo, peso y longitud, se determinó que la mayoría de los individuos eran adultos $(53 \%)$, seguidos por juveniles y preadultos (Cuadro 1). 
Cuadro 2. Detalles del peso y medidas corporales de zarigüeyas capturadas $(n=15)$ en los predios del Zoológico de Cali

\begin{tabular}{|c|c|c|c|c|c|c|c|}
\hline \multirow{2}{*}{$\begin{array}{l}\text { Edad } \\
\text { estimada }\end{array}$} & \multirow{2}{*}{ Sexo } & \multirow{2}{*}{$\begin{array}{c}\text { Peso } \\
(\mathrm{g})\end{array}$} & \multicolumn{4}{|c|}{ Medidas corporales $^{1}(\mathrm{~mm})$} & \multirow{2}{*}{$\begin{array}{l}\mathrm{N} .^{\circ} \mathrm{de} \\
\text { crías }\end{array}$} \\
\hline & & & $\mathrm{LT}$ & $\mathrm{LC}$ & LP & $\mathrm{LO}$ & \\
\hline Juvenil & $\mathrm{M}$ & 830 & 705 & 360 & 40 & 56 & - \\
\hline Juvenil & M & 950 & 790 & 450 & 50 & 45 & - \\
\hline Juvenil & M & 350 & 530 & 280 & 48 & 45 & - \\
\hline Juvenil & $\mathrm{H}$ & 470 & 550 & 310 & 45 & 45 & - \\
\hline Pre-adulto & M & 1850 & 830 & 415 & 63 & 50 & - \\
\hline Pre-adulto & $\mathrm{M}$ & 1690 & 870 & 460 & 63 & 50 & - \\
\hline Pre-adulto & $\mathrm{H}$ & 1550 & 675 & 315 & 50 & 45 & - \\
\hline Adulto & M & 2040 & 820 & 410 & 66 & 50 & - \\
\hline Adulto & M & 2250 & 855 & 440 & 70 & 50 & - \\
\hline Adulto & M & 2070 & 856 & 420 & 65 & 55 & - \\
\hline Adulto & $\mathrm{H}$ & 1830 & 840 & 415 & 60 & 55 & 3 \\
\hline Adulto & $\mathrm{H}$ & 2110 & 835 & 425 & 60 & 62 & 4 \\
\hline Adulto & $\mathrm{H}$ & 1990 & 740 & 395 & 63 & 50 & 4 \\
\hline Adulto & $\mathrm{H}$ & 2015 & 837 & 410 & 65 & 48 & 5 \\
\hline Adulto & $\mathrm{H}$ & 1700 & 930 & 470 & 65 & 60 & 4 \\
\hline
\end{tabular}

${ }^{1}$ LT: Longitud total; LC: Longitud de la cola; LP: Longitud del pie; LO: Longitud de la oreja

El 47\% (n=7) de los animales capturados fueron hembras, donde todas las hembras adultas presentaron crías en el marsupio. Asimismo, se observó un promedio de cuatro crías por hembra adulta (Cuadro 2). Las juveniles y pre-adultas no presentaron crías. Por otro lado, el promedio de peso y longitud total de los machos fue relativamente mayor que el de las hembras.

\section{Discusión}

La familia Didelphidae representa la mayoría de los marsupiales americanos ampliamente distribuidos en América del Sur (Titira, 2007); sin embargo, su distribución geográfica abarca el norte, centro y sur de
América (Emmons y Feer, 1990), ocupando casi todo tipo de hábitats a excepción de zonas muy altas y zonas desérticas (Williams y Genoways, 2008).

Algunos autores describen a miembros de esta familia como medianos y grandes (Cuartas y Muñoz, 2003) y otros como de tamaño pequeño y mediano (Titira, 2007). En el presente estudio se encontraron tres tamaños pero que estuvieron relacionados directamente con el peso: cuatro animales clasificados como juveniles con pesos inferiores a $1000 \mathrm{~g}$, seis animales con pesos entre los 1000 y 2000 g clasificados como preadultos y adultos y cinco animales clasificados como adultos con pesos superiores a $2000 \mathrm{~g}$. Eisenberg (1989) es más detallado en su des- 
cripción respecto al tamaño y manifiesta que existen géneros con animales con $80 \mathrm{~mm}$ entre la cabeza y el cuerpo, hasta géneros con individuos con $1020 \mathrm{~mm}$ desde la cabeza hasta la punta de la cola.

Teniendo en consideración los meses del muestreo y las características climáticas, se podría considerar que la edad de los juveniles y preadultos fue de 4 y 6 meses, respectivamente. Por otro lado, el peso de los individuos adultos se encuentra por encima del promedio dado por Cuartas y Muñoz (2003), que correspondía a $1610 \mathrm{~g}$, pero se encuentra entre los rangos dados por Eisenberg (1989), quien reporta pesos hasta los $5500 \mathrm{~g}$.

En el presente estudio, todas las hembras presentaban marsupio y las hembras adultas estaban con crías. Esto confirma su alta capacidad de reproducción e indica que posiblemente una de las temporadas de apareamiento de las zarigüeyas ocurre entre finales y principios de año, lo cual coincide con la época de baja precipitación y mayor fotoperiodo.

Teniendo en cuenta que el grado de distribución de la población está directamente relacionado con la disponibilidad de alimento, además de otros factores como fuentes de agua y presencia de vegetación arbórea y arbustiva, se podría decir que el territorio utilizado para el muestreo presentaba condiciones medioambientales adecuadas para la presencia de zarigüeyas.

\section{Literatura Citada}

1. Álvarez M, Córdoba S, Escobar F, Fagua G, Gast F, Mendoza H, Ospina M, et al. 2006. Manual de métodos para el desarrollo de inventarios de biodiversidad. Programa de inventarios de Biodiversidad Grupo de Exploración y Monitoreo Ambiental (GEMA). Instituto de Investigación de Recursos Bio- lógicos Alexander von Humbold. Bogotá, Colombia. $236 \mathrm{p}$.

2. Cuartas C, Muñoz J. 2003. Marsupiales, cenoléstidos e insectívoros de Colombia. Medellín: Universidad de Antioquia. $227 \mathrm{p}$.

3. Eisenberg JF. 1989. Mammals of the neotropics. The northern neotropics. Panama, Colombia, Venezuela, Guyana, Suriname, French Guiana. Vol 1. USA: University of Chicago. $550 \mathrm{p}$.

4. Emmons LH, Feer F. 1990. Neotropical rainforest mammals. A field guide. USA: University of Chicago. $281 \mathrm{p}$.

5. Gardner AL, Creighton GK. 2008. Genus Micoureus Lesson, 1842. En: Gardner AL (ed). Mammals of South America. Marsupials, xenarthrans, shrews, and bats. USA: University of Chicago. p 74-82.

6. Grimwood IR. 1969. Notes on the distribution and status of some Peruvian mammals. American Committee for International Wild Life Protection. Monographic Series N. ${ }^{\circ} 21$. New York. $86 \mathrm{p}$.

7. Marques-Aguilar SA. 2008. Genus Artibeus Leach, 1821. En: Gardner AL (ed). Mammals of South America. Marsupials, xenarthrans, shrews, and bats. USA: University of Chicago. p 301321.

8. Oliveira EV, Goin FJ. 2006. Marsupiais do início do terciario do Brasil: origen, irradiçao e história biogeográfica. En: Caceres NC, Monteiro-Filho ELS (eds). Os marsupiais do Brasil: biología, ecología e evoluçao. Campo Grande, Brasil: Universidad Federal do Mato Grosso do Soul. p 607-622.

9. Patton JL, Da Silva MNF, Malcolm JR. 2000. Mammals of the Rio Juruá and the evolutionary and ecological diversification of Amazonia. Bull Am Mus Nat Hist 244: 1-306.

10. Sánchez F. 2004. Inventario de mamíferos en un bosque de los andes centrales de Colombia. Caldasia 26(1): 291-309. 
11. Schweigmann NJ. 1999. Estudio de la prevalencia de la infección por Trypanosoma cruzi en zarigüeyas (Didelphis albiventris) en Santiago de Estero, Argentina. Rev Panam Salud Pública 6: 371-376.

12. Solari S, Vivar E, Velazco PM, Rodriguez JJ. 2001. Small mammals of the southern Vilcabamba region, Peru. En: Alonso LE, Alonso A, Schulenberg TS, Dallmeier F (eds). Biological and social assessments of the Cordillera de Vilcabamba, Peru. RAP Working Papers 12 and SIMAB Series 6. Conservation International. Washington DC. p 110-116.

13. Stein BR, Patton JL. 2008. Genus Lutreolina O. Thomas, 1910. En: Gardner AL (ed). Mammals of South America. Marsupials, xenarthrans, shrews, and bats.
USA: University of Chicago. p 25-27

14. Titira D. 2007. Guía de campo de los mamíferos del Ecuador. $6^{a}$ ed. Quito: Ed Murciélago Blanco. 576 p.

15. Vaughan T, Ryan J, Czaplewski $N$. 1999. Mammalogy. $4^{\text {th }}$ ed. Philadelphia: Saunders College Publishing. 565.

16. Vonhof MJ. 2000. Rhogeessa tumida. Mamm Species 633: 1-3.

17. Williams SL, Genoways HH. 2008. Marsupials. En: Gardner AL (ed). Mammals of South America. Marsupials, xenarthrans, shrews, and bats. USA: University of Chicago. p 255-300.

18. Wilson DE, Reeder DM. 2005. Mammal species of the world. A taxonomic and geographic reference $3^{\text {rd }}$ ed. USA: Johns Hopkins University Press. 2142 p. 